\title{
Who should be treating erectile dysfunction?
}

\author{
Gerald Brock
}

The past decade has been witness to dramatic advances in our understanding of the underlying causes of erectile dysfunction (ED). Armed with this increase in knowledge, improved diagnostic and therapeutic approaches have evolved, allowing almost all men with erectile difficulties to choose some form of effective treatment. The necessity to perform complex invasive diagnostic testing such as cavernosometry or angiography is rare, because it seldom alters management. Pharmacotherapy, the most frequently chosen therapeutic approach for men with ED, is usually noninvasive, in the form of an oral tablet.

Historically, urologists were the physicians of choice in diagnosing and treating impotence. Their ability to surgically manage this condition was unquestioned. In the 1980s, the advent of intracorporal injection of vasoactive agents heralded a new age in the treatment of ED, and the concept of pharmacotherapy was born. In spite of this seemingly attractive approach, few physicians championed the technique and in most western cultures urologists were the group that taught intracorporal injection to their patient population.

Primary care physicians (PCPs) routinely diagnose and treat depression, hypertension, diabetes, benign prostatic hyperplasia and cardiovascular disease. They often have an ongoing relationship with the patient and his partner and, as a result, are likely to have an enhanced understanding of the context within which ED has developed and progressed. Alongside this is their ability to monitor the progression of erectile problems and the success of therapy over time, and to integrate recent information citing erectile difficulties as a potential marker of cardiovascular disease. ....'shared

care'...allows

all healthcare

professionals

to contribute

their

knowledge

and skill

optimally in

order to deliver

appropriate

care.

G Brock is Associate

Professor in the

Department of

Surgery, Division of

Urology at St Joseph

Health Centre in

London, Ontario,

Canada.

\section{Competing interests}

The author declared he has no competing interests.

www.nature.com/clinicalpractice doi:10.1038/ncpuro0188
These are all seemingly strong arguments favoring PCPs as the healthcare providers best positioned to treat ED patients.

If this is the case, why are so few PCPs providing this care? Is a background of urology or sexual medicine necessary for a proactive approach towards ED and sexual medicine? Is the family doctor's inability to test for the underlying causes of ED, or to offer the complete array of treatments, the source of their failure to diagnose and treat the majority of men with ED?

Clearly there is no definitive answer to the question: 'Who, among healthcare providers, is ideally suited to treat ED?' Urologists have expertise in surgical management of ED and perform much of the clinical and basic research in this area, whereas the vast majority of men can be effectively managed by internists and PCPs with the use of oral treatments. The current model of 'shared care', in which large numbers of men are initially treated by their PCP and are only referred on to sexual medicine experts (urologists and others) in cases that are hard to treat, is in widespread use. I believe this is the best way to treat men with ED, and it allows all healthcare professionals to contribute their knowledge and skill optimally in order to deliver appropriate care to those with erectile concerns.

The larger question in my mind is why those treating ED are not treating a higher number of men with such problems. Until healthcare providers initiate a dialog with their patients, and recognize the positive impact that ED therapy has on the quality of life of their patients, and the partners of their patients, more couples will continue to have erectile difficulties than are treated for it. 